\title{
Two landmark class B GPCR structures unveiled
}

High-resolution crystal structures of the human corticotropinreleasing factor $1\left(\mathrm{CRF}_{1}\right)$ receptor and the human glucagon receptor are reported in Nature. These two structures are the first reports of the seventransmembrane domains (TMDs) of the secretin-like group (class B) of G protein-coupled receptors (GPCRs) and reveal important structural details that might aid in the design of modulators of these receptors.

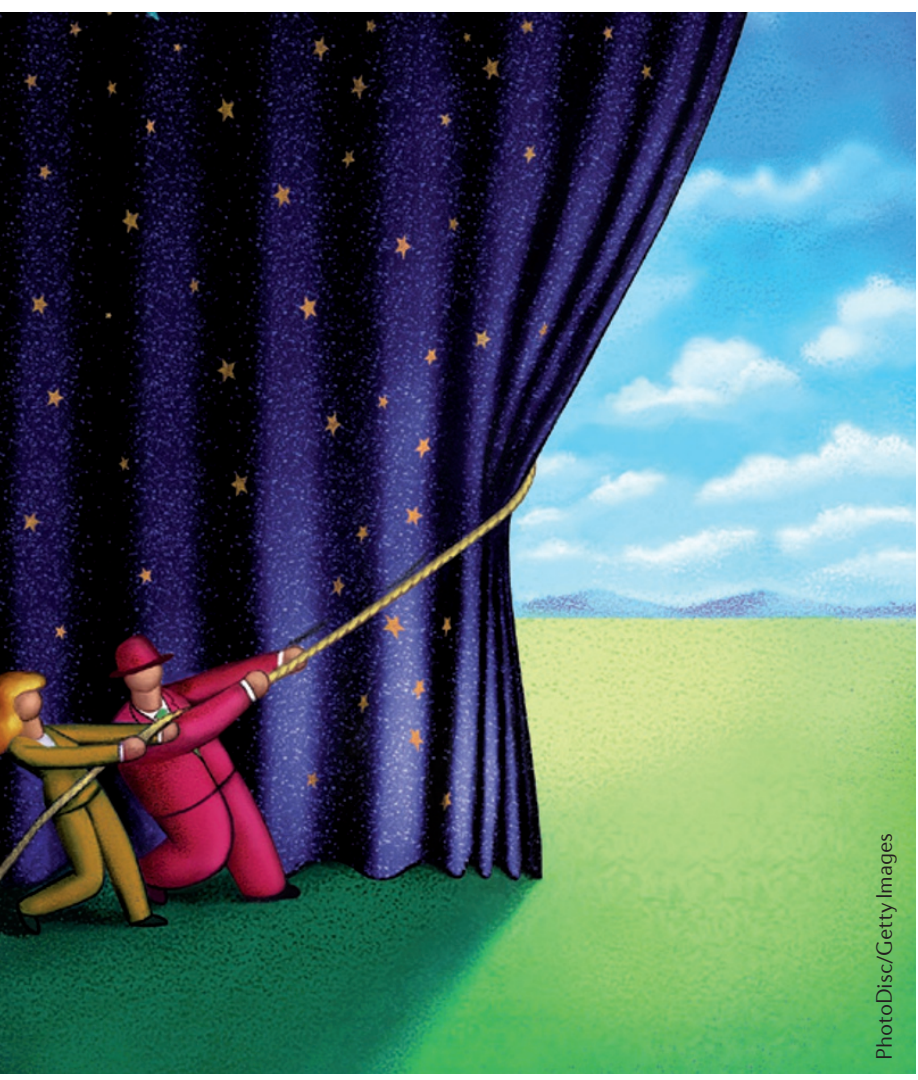

Both structures were resolved in the presence of corresponding antagonists. Although a distinct binding location was identified for the small-molecule antagonist CP-376395 on the $\mathrm{CRF}_{1}$ receptor, no such location was identifiable for the small-molecule antagonist NNC0640 on the glucagon receptor. Nevertheless, the TMD crystal structure of the glucagon receptor combined with the characterization of more than 100 single point mutations and computational modelling of the full-length molecule enabled detailed examination of its peptidebinding site.

For the $\mathrm{CRF}_{1}$ receptor, which is a potential drug target for depression and anxiety, the TMD adopts a pronounced V-shape, in contrast to class A GPCRs. A large cavity is presented on the extracellular side, which the authors suggest is the peptide-binding site. They then compared the $\mathrm{CRF}_{1}$ receptor with the dopamine $\mathrm{D}_{3}$ receptor as an example class A GPCR. Although the two structures show much similarity, striking differences in the TMD were revealed. For example, the extracellular portion of the $\mathrm{CRF}_{1}$ receptor is $\sim 10 \AA$ further away from the TMD than for the $\mathrm{D}_{3}$ receptor.

Analysis of the antagonist-receptor structure showed that CP-376395 binds in a predominantly hydrophobic pocket defined by residues in helix III, helix V and helix VI - away from the large cavity that is presumed to be the peptide-binding site and away from the locations determined with class A GPCRs. The binding location of the antagonist suggests that this may be how it keeps the receptor in the inactive state.

For the glucagon receptor, which is a potential drug target for type 2 diabetes, strong similarities and differences between this receptor and class A GPCRs were also revealed. For example, the orientation and position of the seven helices of the TMD are similar, but the amino terminal in helix I is longer than in any GPCR structure solved so far. The glucagon receptor also has a wider and deeper cavity in the peptide-binding pocket compared with class A GPCRs, similarly to the $\mathrm{CRF}_{1}$ receptor structure.

Extensive mutagenesis, binding and modelling studies yielded detailed information on how peptide ligands bind to their cognate GPCRs. In general, glucagon is shown to form extensive interactions with the extracellular loops of the glucagon receptor and with residues deep within the TMD. Moreover, this model resolves discrepancies in previous efforts to model the binding site location of peptide ligands in class B GPCRs.

Together, these two papers provide a useful basis for the structure-based design of drugs targeting these receptors. Moreover, they provide a platform for resolving full-length receptor-ligand complexes of these and other class B GPCRs.

Man Tsuey Tse

ORIGINAL RESEARCH PAPERS Hollenstein, $\mathrm{K}$. et al. Structure of class B GPCR corticotropinreleasing factor receptor 1. Nature 499, 438-443 (2013)| Siu, F. Y.et al. Structure of the human glucagon class B G-protein-coupled receptor. Nature 499, 444-449 (2013)

FURTHER READING Stevens, R. C. et al. The GPCR Network: a large-scale collaboration to determine human GPCR structure and function. Nature Rev. Drug Discov. 12, 25-34 (2013) 